\title{
O CYBERBULLYING EM CONTEXTO UNIVERSITÁRIO DO BRASIL E PORTUGAL: VITIMIZAÇ̃̃̃O, EMOÇÕES ASSOCIADAS E ESTRATÉGIAS DE ENFRENTAMENTO
}

\author{
EL CYBERBULLYNG EN EL CONTEXTO UNIVERSITARIO DE BRASIL Y PORTUGAL: \\ VICTIMIZACIÓN, EMOCIONES ASOCIADAS Y ESTRATEGIAS DE \\ ENFRENTAMIENTO
}

\begin{abstract}
THE CYBERBULLYING IN CONTEXT UNIVERSITY OF BRAZIL AND PORTUGAL: VICTIMIZATION, EMOTIONS ASSOCIATES AND COPING STRATEGIES
\end{abstract}

\author{
Sidclay Bezerra de SOUZA ${ }^{1}$ \\ Ana Margarida VEIGA SIMÃO ${ }^{2}$ \\ Paula da Costa FERREIRA ${ }^{3}$ \\ Paula PAULINO ${ }^{4}$ \\ Sofia Mateus FRANCISCO ${ }^{5}$
}

RESUMO: O presente artigo buscou, por um lado, descrever como os estudantes universitários do Brasil e de Portugal experienciaram situações de cyberbullying no papel de vítimas, considerando-se algumas variáveis sociodemográficas, bem como analisar se existiam diferenças significativas em função do contexto relativamente aos comportamentos de vitimização, as emoções e as estratégias utilizadas pelas vítimas do Brasil e de Portugal. Buscando atender aos objetivos da investigação, 1340 estudantes universitários (Brasil: $n=592$; Portugal: $n=748$ ) responderam ao Questionário do Cyberbullying para o Ensino Superior (QCES). As análises realizadas permitiram verificar que uma percentagem de $44.6 \%$ dos participantes brasileiros e $43.0 \%$ dos participantes portugueses foram vítimas de cyberbullying em algum momento da vida e algumas diferenças foram encontradas no que diz respeito aos comportamentos de vitimização, as emoções das vítimas e as estratégias de enfrentamento utilizadas pelas vítimas de cada país. Os resultados são discutidos, algumas limitações são consideradas e são apresentadas algumas implicações que sugerem a importância do desenvolvimento sistêmico de programas de prevenção e intervenção nos contextos universitários.

PALAVRAS-CHAVE: Cyberbullying. Estudantes universitários. Vitimização. Emoções. Estratégias de enfrentamento.

${ }^{1}$ Doutor em Psicologia, Especialidade em Psicologia da Educação pela Faculdade de Psicologia Universidade de Lisboa, Lisboa - Portugal, Email: ssouza@campus.ul.pt;

2 Professora Associada com Agregação da Faculdade de Psicologia - Universidade de Lisboa, Lisboa Portugal, Email: amsimao@psicologia.ulisboa.pt;

3 Pós-doutoranda em Psicologia, Especialidade em Psicologia da Educação pela Faculdade de Psicologia - Universidade de Lisboa, Lisboa - Portugal; Email: paula.costa.ferreira@gmail.com;

4 Doutora em Psicologia, Especialidade em Psicologia da Educação pela Faculdade de Psicologia Universidade de Lisboa, Lisboa - Portugal; Email: a.paula.paulino@gmail.com;

5 Doutoranda em Psicologia, Especialidade em Psicologia da Educação pela Faculdade de Psicologia Universidade de Lisboa, Lisboa - Portugal, Email:sofifrancisco@gmail.com. 
RESUMEN: El presente artículo busco, por un lado, describir como los estudiantes universitarios de Brasil y Portugal experimentaron situaciones de cyberbullying en el papel de víctimas, considerándose algunas variables sociodemográficas, bien como analizar si existían diferencias significativas en función del contexto relativo a los comportamientos de victimización, las emociones y las estrategias utilizadas por las victimas de Brasil y Portugal. Buscando atender a los objetivos de la investigación, 1340 estudiantes universitarios (Brasil: $n=592$; Portugal: $n=748$ ) respondieron el cuestionario de Cyberbullying para enseñanza superior (QCES). Los análisis realizados permitieron verificar que un porcentaje de $44.6 \%$ de los participantes brasileros y $43 \%$ de los participantes portugueses fueron víctimas de cyberbullying en algún momento de la vida, y algunas diferencias fueron encontradas respecto a los comportamientos de victimización, las emociones de las víctimas y las estrategias de enfrentamiento utilizadas en cada país. Los resultados son discutidos, algunas limitaciones son consideradas e son presentadas implicancias que sugieren la importancia del desenvolvimiento sistémico de programas de prevención e intervención en los contextos universitarios.

PALABRAS CLAVE: Cyberbullying. Estudiantes universitarios. Victimización. Emociones. Estrategias de enfrentamiento.

ABSTRACT: The present article searched to describe how the university students of Portugal and Brazil they experienced the role of be victims in cyberbullying considering some sociodemographic variables as well as depending on the context in relation to the conduct of victimization, the emotions and the strategies used by victims of Brazil and Portugal students. Seeking to meet the objectives of the investigation, 1340 students (Brazil: $n=592$; Portugal: $n=748$ ) responded to the Cyberbullying Inventory for College Students (CICS). The analyzes allowed to verify that a percentage of $44.6 \%$ of participants from Brazil and $43.0 \%$ of participants from Portugal were victims of cyberbullying at some point in life and some differences were found with regard to the conduct of victimization, the emotions of victims and the coping strategies used by victims of each country. The results are discussed, some limitations are considered and are some implications that suggest the importance of systemic development of prevention and intervention programs in university contexts.

KEYWORDS: Cyberbullying. College Students. Victimization. Emotions. Coping strategies.

\section{Introdução}

O uso extensivo das tecnologias da informação e comunicação (TIC), incluindo a internet e os telefones móveis, transformou a atmosfera da aprendizagem tradicional num mundo cibernético em que os alunos podem obter novas vivências e sentimentos (SMITH et al., 2008). Apesar das inúmeras vantagens resultantes das rápidas mudanças 
tecnológicas, o paradigma tecnológico se difundiu trazendo algumas repercussões aos seus utilizadores considerando os efeitos dos usos sociais da própria tecnologia (CASTELLS, 2006). Neste cenário, mencionamos a problemática do cyberbullying, considerado como um problema mundial grave.

O cyberbullying é caracterizado como uma nova expressão do bullying enquanto agressão, ameaça e provocação de desconforto, sendo premeditadas e repetidas, realizadas com recurso a dispositivos tecnológicos de comunicação (e.g., e-mail, chat, blogue, telefones móveis, etc.), contra uma vítima de estatuto semelhante, mas que tem dificuldade em defender-se (PESSOA; MATOS; AMADO; JÄGER, 2011), uma vez que o agressor vive em busca de um prazer ou lucro através dos maus tratos de outro indivíduo ou um grupo por meio das tecnologias (HINDUJA; PATCHIN, 2007). Suas manifestações são realizadas através da difusão de informações eletrônicas de forma prejudicial e difamatória através de emails, mensagens instantâneas, mensagens de texto através de telefones móveis, como ainda, através de publicação de vídeos e/ou fotografias em sites e redes sociais (GARAIGORDOBIL, 2011).Como descrito por Francisco, Veiga Simão, Ferreira e Martins (2015), os comportamentos observados de cyberbullying incluem atitudes de intimidação (e.g., ameaçar, assediar, insultar, espalhar rumores, passar-se por outro estudante), como também de apropriação de imagem (e.g., usar a imagem sem a autorização).

A cada dia, constatamos um número crescente de estudos sobre o cyberbullying com estudantes do ensino superior em diversos Países (e.g., ABOUJAOUDE; SAVAGE, STARCEVIC; SALAME, 2015; SOUZA; VEIGA SIMÃO; CAETANO, 2014a; FRANCISCO et al., 2015). Neste sentido, derivado dos trágicos incidentes e dos crescentes problemas de cyberbullying nas universidades, as instituições estão se esforçando para compreender o impacto do fenômeno entre os estudantes universitários (WASHINGTON, 2015).

O cyberbullying está a tornar-se um problema social grave que tem preocupado os investigadores e educadores em termos internacionais (LI, 2008), por ser praticado onde quer que o agressor disponha de recursos necessários, seja nos contextos educacionais, em casa ou na rua (PESSOA; MATOS; AMADO; JÄGER, 2011) de forma a produzir danos irreparáveis na vida das vítimas. Estudos evidenciam que o cyberbullying está associado com o aumento de problemas psicossociais (FAUCHER; JACKSON; CASSIDY, 2014; OLWEUS, 2012), baixo compromisso dos estudantes (YBARRA; MITCHELL, 2004), com o consumo de álcool (CAPPADOCIA; CRAIG; 
PEPLER, 2013), sendo ainda identificados problemas psiquiátricos relativamente ao alto nível da ansiedade, da depressão e da ideação suicida (SCHENK; FREMOUW, 2012).

\section{Das Emoções às Estratégias de Enfrentamento em Situações de Cyberbullying}

Atualmente, com o avanço das investigações sobre o cyberbullying, investigadores de toda parte do mundo têm somado esforços no sentido de compreender as questões envolvidas em situações de cyberbullying. Alguns estudos têm procurado compreender a questão das emoções derivadas de situações de cyberbullying e do bullying presencial, considerando os tipos de envolvimento (GUALDO; HUNTER; DURKIN; ARNAIZ; MAQUILÓN, 2015; ORTEGA et al., 2012). Como argumenta Lazarus (1991), as emoções dependem da avaliação cognitiva dos eventos e envolvem os aspectos relacionais, motivacionais e cognitivos. Além disso, as interações sociais que os estudantes estabelecem são muitas vezes acompanhadas por uma intensa experiência emocional (BAHIA; FREIRE, AMARAL; ESTRELA, 2013), considerando o papel que desempenham nas relações (GUALDO et al., 2015).

Os estudos sobre as emoções como (re)ações às situações de cyberbullying são importantes (WILTON; CAMPBELL, 2011) visto que a competência emocional é uma parte essencial do bem-estar pessoal e social dos estudantes (BAHIA et al., 2013).Neste sentido, o estudo conduzido por Gualdo et al., (2015), revelou que a reação emocional mais comum que os agressores esperam das vítimas foi o medo, seguido pelo sentimento de ofensa. Em contraste, as vítimas de cyberbullying relataram que a emoção mais intensa foi a tristeza, seguido do sentimento de rejeição. No estudo realizado por Caetano, Freire, Veiga Simão, Martins e Pessoa (2016), constatou-se que a tristeza, a vontade de vingança e o medo são as emoções mais frequentes das vítimas, enquanto a satisfação, a indiferença e o alívio são aquelas que os agressores mais vivenciam. De acordo com os autores, os resultados sugerem a necessidade do desenvolvimento de uma educação emocional dos jovens como uma das formas de prevenção do cyberbullying.

Outros estudos têm buscado compreender a questão da utilização das estratégias de enfrentamento em situações de cyberbullying. Castilho (2010) considera as 
estratégias de enfrentamento como o manejo de esforços cognitivos e comportamentais para lidar com demandas específicas e podem ser alteradas em função das situações desencadeadoras (CASTAÑO; BARCO, 2010). Sob esta perspectiva, Price e Dalgleish (2010) consideram que as estratégias utilizadas na resolução de situações de cyberbullying podem ser tomadas tanto individualmente como por amigos dos envolvidos, quer pela comunidade educativa, como também, pelos pais ou encarregados de educação.

No estudo desenvolvido por Souza et al., (2014a) com estudantes universitários portugueses, verificou-se que fazer frente ao fato, contactar as autoridades policiais, procurar ajuda de alguém de confiança e pedir ajuda aos amigos, foram as estratégias offline mais reportadas pelas vítimas. Além disso, restringir e encerrar contatos, excluir os agressores das redes sociais e contatar os gestores do site ou rede social foram indicadas com maior frequência, como estratégias online. A atual literatura sobre as estratégias de enfrentamento em situações de cyberbullying reforça o papel do suporte social quando as vítimas não podem defender-se facilmente. Por exemplo, o estudo de Ševčíková, Macháčková, Wright, Dědková e Černá (2015), revelou que as vítimas que apresentam fracas relações tanto com os seus pares como com os seus pais, foram menos propensas a pedirem ajuda aos outros.

Tendo como ponto de partida os aspectos referidos seja em termos da relevância dos estudos com os estudantes universitários, bem como a importância dos estudos sobre as emoções e as estratégias de enfrentamento, definimos os seguintes objetivos: a)descrever como os estudantes universitários do Brasil e de Portugal experienciaram situações de cyberbullying no papel de vítimas, considerando as variáveis gênero, faixaetária, ano de escolaridade e área científica; b) analisar se existiam diferenças significativas em função do contexto relativamente aos comportamentos de vitimização, as emoções associadas e as estratégias de enfretamento utilizadas pelas vítimas do Brasil e de Portugal.

\section{Metodologia}

\section{Instrumento}

Utilizamos o Questionário do Cyberbullying para o Ensino Superior (QCES) e a sua versão adaptada para o contexto universitário do Brasil (QCES-Br). O QCES foi 
construído pela equipe de investigação do Projeto Cyberbullying: Um diagnóstico da Situação em Portugal ${ }^{2}$. Trata-se de um instrumento de autorrelato com 4 escalas, sendo cada uma delas composta por um conjunto de nove itens com três opções de respostas em escala de tipo Likert ( 1 = Nunca; 2 = Algumas Vezes e $3=$ Muitas Vezes). Uma escala é destinada às vítimas, outra aos agressores, outra aos observadores das vítimas e uma aos observadores dos agressores. Estudos foram realizados com ambas as versões do instrumento, que se encontram validadas para utilização seja no contexto universitário de Portugal como do Brasil (AZEVEDO, 2013; FRANCISCO, 2012; FRANCISCO et al., 2015; FERREIRA et al., 2016; SOUZA, 2011; SOUZA et al, 2014a; SOUZA, 2016).

\section{Participantes}

Contamos com a participação de um total de 1340 estudantes universitários. A caracterização dos participantes de cada país é apresentada na Tabela 1.

Tabela 1. Caracterização dos Participantes por País

\begin{tabular}{lcc}
\hline & $\begin{array}{c}\text { Brasil } \\
(n=592)\end{array}$ & $\begin{array}{c}\text { Portugal } \\
(n=781)\end{array}$ \\
\hline Gênero & & \\
$\quad$ Masculino & 34.5 & 33.8 \\
$\quad$ Feminino & 65.5 & 66.2 \\
Faixa-etária & & \\
$<20$ anos & 53.7 & 61.9 \\
$21-23$ anos & 26.4 & 28.5 \\
$24-26$ anos & 9.8 & 3.5 \\
26 anos & 10.1 & 6.1 \\
Area Científica & & 12.4 \\
Ciências da Saúde e da Vida & 22.0 & 19.8 \\
Ciências Exatas e Engenharia & 19.1 & 28.7 \\
Ciências Naturais e do Meio Ambiente & 23.5 & 39.0 \\
Ciências Sociais e Humanidades & 35.5 & \\
Ano de Escolaridade & & 38.0 \\
1. ${ }^{\circ}$ ano do ensino superior & 35.1 & 28.2 \\
2. ${ }^{\circ}$ ano do ensino superior & 31.4 & 33.8 \\
3. ano do ensino superior & 33.4 & \\
\hline
\end{tabular}

Nota. Os valores apresentados nesta tabela tratam-se de \%.

\section{Procedimento de Recolha e Análise dos Dados}

A aplicação dos instrumentos aconteceu em contexto de sala de aula, após a obtenção do consentimento oral por parte dos Professores e Coordenadores dos Cursos e

${ }^{2}$ O projeto foi aprovado pela Fundação para a Ciência e Tecnologia (FCT) com a referência: PTDC/CPECED/108563/2008, financiado pelo Programa Operacional Temático Fatores de Competitividade (COMPETE) e comparticipado pelo Fundo Europeu de Desenvolvimento Regional (FEDER). 
dos estudantes. Os participantes, em ambos os contextos, foram informados acerca dos objetivos do estudo e foram assegurados da confidencialidade dos dados e da preservação da identidade dos mesmos, podendo ser interrompida pelos participantes em qualquer momento. $\mathrm{Na}$ fase de recolha de dados, foi disponibilizado aos participantes a presença de um psicólogo clínico como apoio (FRANCISCO et al., 2015; FERREIRA et al., 2016; SOUZA et al., 2014a).

Os dados foram analizados através do software IBM-SPSS 22.0 para a realização das análises. Considerando o tamanho da amostra e as variáveis estudadas, utilizamos o $t$-teste para igualdade das variâncias objetivando verificar a existência de diferenças significativas entre os Países. Os resultados marginalmente significativos foram considerados (MARTINS, 2011).

\section{Resultados}

Da totalidade dos participantes de cada país, verificamos que 246 dos estudantes universitários brasileiros (representando uma percentagem de 44.6\%) e 322 estudantes universitários portugueses (representando uma percentagem de $43.0 \%$ ) foram vítimas de cyberbullying em algum momento da vida. Em ambos os Países, a maior percentagem das vítimas era do gênero feminino, tinham idade igual ou inferior aos 20 anos, eram da área de Ciências Sociais e Humanidades e frequentavam o $1 .^{\circ}$ ano do ensino superior (ver Tabela 2).

Tabela 2. Gênero, Faixa-etária, Área Científica e Ano de Escolaridade das Vítimas por País

\begin{tabular}{lcc}
\hline & $\begin{array}{c}\text { Brasil } \\
(n=246)\end{array}$ & $\begin{array}{c}\text { Portugal } \\
(n=322)\end{array}$ \\
\hline Gênero & & \\
$\quad$ Masculino & 31.3 & 32.6 \\
$\quad$ Feminino & 68.7 & 67.4 \\
Faixa-etária & & \\
$\geq 20$ anos & 58.9 & 62.5 \\
$21-23$ anos & 24.4 & 30.7 \\
$24-26$ anos & 8.9 & 3.4 \\
26 anos & 7.8 & 3.4 \\
Área Científica & & \\
Ciências da Saúde e da Vida & 25.2 & 9.9 \\
Ciências Exatas e da Engenharia & 12.6 & 23.0 \\
Ciências Naturais e do Meio Ambiente & 26.4 & 28.6 \\
Ciências Sociais e Humanidades & 35.8 & 38.5 \\
Ano de Escolaridade & & \\
1. ano do Ensino Superior & 36.2 & 38.2
\end{tabular}


2. ${ }^{\circ}$ ano do Ensino Superior

Nota. Os valores apresentados na tabela tratam-se de $\%$.

Na sequência, analisamos a existência de diferenças significativas relativamente aos comportamentos experienciados pelas vítimas, as emoções referidas pelas vítimas e as estratégias utilizadas pelas vítimas do Brasil e de Portugal. Conforme Tabela 3, observamos que, as vítimas do Brasil tendem a ser mais alvos de ameaças $[t(556)=-$ $2.22, p=.026]$ e assédios com conteúdos de caráter sexual $[t(556)=-1.70, p=.088]$ que as vítimas de Portugal.

Tabela 2. Estatística Descritiva e Diferenças entre Brasil e Portugal quanto aos Comportamentos de Vitimização

\begin{tabular}{lccc}
\hline \multirow{2}{*}{ Comportamentos } & Brasil & Portugal & \multirow{2}{*}{$\boldsymbol{t}(\mathbf{5 6 6})$} \\
\cline { 2 - 3 } & $\boldsymbol{M}(\boldsymbol{D P})$ & $\boldsymbol{M}(\boldsymbol{D P})$ & \\
\hline Ameaçaram-me & $1.19(.42)$ & $1.28(.52)$ & $-2.22^{*}$ \\
Assediaram-me com conteúdos de caráter sexual & $1.26(.49)$ & $1.34(.54)$ & -1.701 \\
Espalharam boatos sobre a minha vida & $1.55(.58)$ & $1.56(.58)$ & -.12 \\
Fizeram-se passar por mim & $1.24(.44)$ & $1.27(.49)$ & -.57 \\
Zombaram-me & $1.62(.60)$ & $1.58(.61)$ & .81 \\
Insultaram-me & $1.52(.60)$ & $1.58(.60)$ & -1.08 \\
Mostraram que possuíam informação sobre a minha vida & $1.30(.53)$ & $1.27(.50)$ & -70 \\
que podem afetar o meu bem-estar psicológico & & & \\
Revelaram dados sobre a minha vida privada & $1.26(.47)$ & $1.26(.47)$ & -.14 \\
Usaram a minha imagem sem autorização & $1.37(.51)$ & $1.34(.51)$ & .62 \\
\hline
\end{tabular}

Nota. $1<.10 .{ }^{*} p<.05 ;$ Brasil = 1; Portugal = 2; Brasil $(n=246) ;$ Portugal $(n=322)$.

Conforme Tabela 4, verificamos que as vítimas do Brasil tendem, significativamente, a sentir mais culpa $[t(556)=-2.05, p=.041]$, preocupação $[t(556)$ $=-2.85, p=.005]$, indefesos $[t(556)=-2.51, p=.000]$, raiva $[t(556)=-5.68, p=.041]$, surpresa $[t(556)=-3.27, p=.001]$ e vontade de vingança $[t(556)=-2.26, p=.024]$ que as vítimas de Portugal. Ainda verificamos uma diferença marginalmente significativa no que diz respeito a humilhação. Ou seja, as vítimas do Brasil tendem a sentir-se mais humilhadas $[t(556)=-1.91, p=.056]$ que as vítimas de Portugal. 
Tabela 4. Estatística Descritiva e Diferenças entre as Vítimas do Brasil e Portugal quanto as Emoções

\begin{tabular}{lrrr}
\hline \multirow{2}{*}{ Emoções } & Brasil & Portugal & \multirow{2}{*}{$\boldsymbol{t}(\mathbf{5 6 6 )}$} \\
\cline { 2 - 3 } & $\boldsymbol{M ( \boldsymbol { D P } )}$ & $\boldsymbol{M}(\boldsymbol{D P})$ & \\
\hline Alegria & $1.98(.14)$ & $1.96(.19)$ & 1.35 \\
Ciúme & $1.98(.14)$ & $1.99(.07)$ & -1.51 \\
Culpa & $1.91(.29)$ & $1.95(.21)$ & $-2.05^{*}$ \\
Desprezo & $1.78(.41)$ & $1.80(.39)$ & -.57 \\
Embaraço & $1.74(.44)$ & $1.73(.44)$ & .26 \\
Indiferença & $1.70(.45)$ & $1.75(.43)$ & -1.37 \\
Injustiça & $1.74(.44)$ & $1.75(.43)$ & -.42 \\
Confuso e perdido & $1.79(.40)$ & $1.84(.36)$ & -1.62 \\
Inferioridade & $1.78(.41)$ & $1.79(.41)$ & -.26 \\
Insegurança & $1.65(.47)$ & $1.65(.47)$ & .03 \\
Inveja & $1.98(.14)$ & $1.98(.12)$ & -.43 \\
Medo & $1.76(.42)$ & $1.78(.41)$ & -.51 \\
Orgulho & $1.98(.12)$ & $1.97(.17)$ & 1.12 \\
Preocupação & $1.62(.48)$ & $1.73(.44)$ & $-2.85^{* *}$ \\
Humilhação & $1.68(.46)$ & $1.75(.43)$ & -1.911 \\
Indefeso & $1.74(.44)$ & $1.82(.38)$ & $-2.51^{*}$ \\
Raiva & $1.48(.50)$ & $1.70(.45)$ & $-5.68 * * *$ \\
Superioridade & $1.96(.20)$ & $1.93(.24)$ & 1.04 \\
Surpresa & $1.80(.39)$ & $1.90(.30)$ & $-3.27 * *$ \\
Tristeza & $1.67(.47)$ & $1.72(.44)$ & -1.28 \\
Desespero & $1.88(.32)$ & $1.89(.30)$ & -.61 \\
Aterrorizado & $1.88(.32)$ & $1.91(.28)$ & -1.36 \\
Vontade de me vingar & $1.78(.41)$ & $1.86(.35)$ & $-2.26^{* * *}$ \\
Sozinho/isolado & $1.81(.39)$ & $1.85(.35)$ & -1.20 \\
\hline
\end{tabular}

Nota. $1<.10 .{ }^{*} p<.05 ;{ }^{* *} p<.01 ;{ }^{* * * *} p<.001 ;$ Brasil = 1; Portugal $=2$; Brasil $(n=246)$; Portugal $(n=322) ;$ Assinala $=1 ;$ Não Assinala $=2$.

Relativamente as estratégias de enfrentamento utilizadas pelos estudantes do Brasil e de Portugal, verificamos que as vítimas do Brasil referem confrontar mais os agressores $[t(556)=3.64, p<.001]$, ignorar a agressão [ $t(556)=-2.45, p=.015]$, e que tendem mais o contato com o agressor $[t(556)=-2.12, p=.034]$. Em contrapartida, as vítimas de Portugal referem mais que as vítimas do Brasil que deixaram de atender chamadas anônimas $[t(556)=3.54, p<.001]$ e que agrediram fisicamente os agressores $[t(556)=2.64, p=.009]$. Ainda percebemos uma diferença marginalmente significativa com relação a procurar ajuda profissional $[t(556)=-1.83, p=.067]$. Ou seja, as vítimas do Brasil referem que procuram mais ajuda profissional que as vítimas de Portugal. 
Tabela 6. Estatística Descritiva e Diferenças entre as Vítimas do Brasil e Portugal quanto as Estratégias de Enfrentamento Utilizadas pelas Vítimas

\begin{tabular}{lccc}
\hline \multirow{2}{*}{ Estratégias } & Brasil & Portugal & \multirow{2}{*}{$\boldsymbol{t}(\mathbf{5 6 6})$} \\
\cline { 2 - 3 } & $\boldsymbol{M}(\boldsymbol{D P})$ & $\boldsymbol{M}(\boldsymbol{D P})$ & \\
\hline Alterei o perfil de privacidade & $1.67(.47)$ & $1.71(.45)$ & -.98 \\
Apaguei a página da rede social & $1.91(.28)$ & $1.92(.19)$ & -.20 \\
Confrontei o agressor & $1.80(.39)$ & $1.64(.48)$ & $3.64^{* * *}$ \\
Contactei o gestor do site & $1.94(.24)$ & $1.94(.22)$ & -.30 \\
Desativei o e-mail & $1.94(.23)$ & $1.95(.21)$ & -.45 \\
Tomei mais atenção à webcam do computador & $1.96(.19)$ & $1.97(.15)$ & -.81 \\
Exclui o agressor da rede social & $1.62(.48)$ & $1.69(.46)$ & -1.58 \\
Ignorei a agressão & $1.67(.47)$ & $1.78(.41)$ & $-2.45^{*}$ \\
Apaguei as mensagens (e.g., fotos, vídeos, etc) & $1.77(.42)$ & $1.81(.39)$ & -1.01 \\
Tentei descobrir quem era o agressor & $1.86(.34)$ & $1.86(.34)$ & -.16 \\
Fiz-lhe o mesmo & $1.98(.12)$ & $1.97(.15)$ & .58 \\
Mudei de número de telefone móvel & $1.95(.20)$ & $1.96(.20)$ & -1.39 \\
Deixei de atender chamadas anônimas & $1.92(.27)$ & $1.80(.40)$ & $3.54^{* * *}$ \\
Evitei o contacto com o agressor & $1.65(.47)$ & $1.75(.43)$ & $-2.12^{*}$ \\
Contactei a polícia & $1.97(.16)$ & $1.95(.21)$ & .95 \\
Contei aos meus pais & $1.83(.37)$ & $1.82(.38)$ & .22 \\
Contei aos meus amigos & $1.71(.45)$ & $1.69(.46)$ & .43 \\
Contei aos meus professores & $1.90(.29)$ & $1.94(.23)$ & -1.40 \\
Procurei apoio profissional & $1.93(.25)$ & $1.97(.17)$ & -1.831 \\
Agredi fisicamente quem me fez isso & $2.00(.00)$ & $1.96(.19)$ & $2.64^{* * *}$ \\
\hline Nota.t< & &
\end{tabular}

Nota. $1<.10 .{ }^{*} p<.05 ;{ }^{* * *} p<.01 ;{ }^{* * *} p<.001 ;$ Brasil = 1; Portugal = 2; Brasil $(n=246)$; Portugal $(n=322)$; Assinala $=1 ;$ Não Assinala $=2$.

\section{Discussão dos Resultados}

O presente estudo buscou descrever como os estudantes universitários do Brasil e de Portugal experienciaram situações de cyberbullying no papel de vítimas, considerando-se o gênero, idade, área científica e ano de escolaridade), como também, analisar se existiam diferenças significativas em função do contexto relativamente aos comportamentos de vitimização, as emoções e as estratégias utilizadas pelas vítimas do Brasil e de Portugal.

De modo geral, evidenciamos que cerca de $44.6 \%$ dos estudantes universitários brasileiros e $43.0 \%$ dos universitários portugueses foram vítimas de cyberbullying, sendo as vítimas, maioritariamente do gênero feminino, com idade igual ou inferior aos 20 anos, da área de Ciências Sociais e Humanidades e estudantes do $1 .^{\circ}$ ano do ensino superior. Os resultados obtidos vão ao encontro de outros estudos com estudantes do ensino superior que verificaram a prevalência relativamente ao gênero (LINDSAY; KRYSIK, 2012; SCHENK; FREMOUW, 2012) e idade (e.g., FRANCISCO et al., 2015; GARAIGORDOBIL, 2015). Estes resultados confirmam o que se verifica em outros 
estudos em que as taxas de prevalência entre os estudantes universitários variam muito, embora se verifique que uma parcela substancial dos estudantes universitários vivencia situações de cyberbullying (DILMAC, 2009; DOANE; PEARSON; KELLEY, 2014). Com relação as ocorrências de acordo com a área científica, acreditamos que este estudo fornece informações que permitem ter uma ideia sobre as experiências de cyberbullying dos estudantes das áreas científicas do Brasil e de Portugal.

Visto a importância de investigações sobre o cyberbullying numa perspectiva intercultural (BAEK; BULLOCK, 2014), verificamos diferenças significativas entre os estudantes universitários do Brasil e de Portugal relativamente aos comportamentos experienciados, as emoções relatadas pelas vítimas e as estratégias utilizadas pelas vítimas do Brasil e de Portugal. Os resultados obtidos apresentados neste estudo no que diz respeito as diferenças verificadas em termos dos comportamentos de vitimização experienciados nos permitem concordar com Crystal (1994), ao referir que a prevalência e o significado de qualquer um desses comportamentos variam de um contexto cultural para outro. Tal fato, pode ser justificado, por exemplo, pelo nível de individualismocoletivismo que cada país apresenta (HOFSTEDE, 2001) que está relacionado com a agressão direta e indireta (FORBES; ZHANG; DOROSZEWICZ; HAAS, 2009), como ainda pelo papel que a cultura desempenha nos comportamentos agressivos dos estudantes (BAEK; BULLOCK, 2014).

As diferenças verificadas neste estudo em termos das emoções associadas ao cyberbullying, bem como acerca das estratégias de enfrentamento nos permitem compreender que ambas podem variar em função do contexto cultural (BAEK; BULLOCK, 2014; LI, 2008). Relativamente as emoções, os nossos resultados vão ao encontro de ouros estudos que verificaram que as vítimas experimentam emoções negativas como frustração, raiva, desesperança e tristeza (NA; DANCY; PARK, 2015; CAETANO et al., 2016). Além disso, no que diz respeito às estratégias de enfrentamento utilizadas, os resultados apresentados nos permitem perceber como os estudantes do Brasil e de Portugal que foram vítimas lidam com as situações de cyberbullying. Ou seja, as vítimas do Brasil apresentam uma tendência maior para o confronto ou até mesmo ignorar o agressor, como ainda buscar ajuda profissional, ao passo que as vítimas de Portugal apresentaram uma maior tendência para estratégias mais extremas, utilizando por sua vez estratégias de evitamento a medida em que deixam de atender as chamadas dos agressores ou até mesmo de confronto direto ao agressor. 
De qualquer modo, o cyberbullying enquanto um tipo de agressão relacional que ocorre através dos dispositivos tecnológicos (GARAIGORDOBIL, 2011), está associado com a saúde subjetiva dos estudantes, cujas repercussões podem ser incalculáveis na vida dos envolvidos (SOUZA et al., 2014a; WASHINGTON, 2015). Este impacto não se relaciona apenas com as vítimas. A segurança e bem-estar dos agressores também deverá ser uma preocupação, sendo necessário intervir para auxiliar na mudança do seu comportamento (FRANCISCO, 2012).

\section{Limitações e Futuros Estudos}

Após uma breve discussão dos resultados globais deste estudo é importante ter em consideração algumas limitações que lhe estão inerentes. Inicialmente, há de se referir que uma das limitações deste estudo diz respeito ao número de participantes. $\mathrm{Ou}$ seja, os resultados apresentados não podem ser generalizados. Em termos de futuras investigações, seria interessante obter um número de participantes mais elevado e de diversas instituições universitárias do Brasil e de Portugal, permitindo uma maior representatividade da população de ambos os países.

Acreditamos na importância de estudos futuros que objetivem compreender como as vítimas conseguem lidar com situações de cyberbullying e ser resilientes, ao invés de reproduzirem o mesmo comportamento. Por fim, destacamos a importância de estudos que busquem analisar a questão da regulação socioemocional que permitam prevenir situações de cyberbullying e ensinar aos estudantes a aprender a lidar com o problema, ao invés de reproduzir o comportamento de agressão online.

\section{Implicações para a Prática}

Em termos de implicações, considera-se necessário informar e formar os estudantes, advertir sobre os perigos das tecnologias, ensinar estratégias de enfrentamento e trabalhar competências sociais, essenciais para o desenvolvimento positivo dos jovens (SOUZA et al., 2014b; FRANCISCO, 2012) e que os convidem a assumir compromissos mais significativos nos relacionamentos da vida real. Ao mesmo tempo, não podemos deixar de referir a importância da criação de comunidades educacionais que proporcionem aos estudantes a consciência do respeito, compromisso e responsabilidade (BERKENBROCK-ROSITO; JOSÉ, 2015). Os resultados apresentados neste artigo são relevantes por permitirem às intuições universitárias 
obterem informações sobre o que pode ser feito em termos de prevenção e intervenção (FERREIRA et al., 2016; SOUZA et al., 2014b). Neste sentido, é necessário a realização de estudos que possibilitem discenir quais as estratégias de enfrentamento que promovam um melhor ajuste psicológico aos estudantes universitários (NA et al., 2015), ao invés de lidar com a situação com comportamentos capazes de continuar o ciclo da agressão.

Martins (2009) sugere a importância das estratégias pedagógicas na prevenção deste tipo de problemas, tais como a resolução de conflitos de forma não violenta, a discussão de dilemas hipotéticos e da vida real, a dramatização e representação de papéis, a promoção da assertividade e das competências da comunicação social. Referimos também a importância do uso de estratégias de prevenção que contemplem a promoção do desenvolvimento de competências socioemocionais, que promovam o auto autoconhecimento, a consciência social, a capacidade de resolver conflitos e que permitam lidar com diferentes situações ao longo da vida. Trata-se de promover o desenvolvimento de comunidades educativas, como assinalado por Souza et al., (2014a), onde todos se sintam parte do problema, autorizados para intervir com responsabilidade, e desenvolver uma sociedade, uma comunidade e um contexto de aprendizagem inclusivo, eticamente justo e cuidador.

\section{Considerações Finais}

O cyberbullying enquanto uma forma de violência, trata-se de uma construção coletiva e que permeia todas as culturas. Um fenômeno de dinâmica grupal (CASTILHO, 2010), marcado pela expressão do sentimento de intolerância de um ou mais indivíduos direcionado ao(s) outro(s) que é influenciado pelo contex to em que este grupo se encontra inserido (SOUZA, 2016). De todo modo, a violência que acontece nos contextos educacionais deve ser vista como um algo que acontece de forma "velada" e opera no campo da cultura (BERKENBROCK-ROSITO; JOSÉ, 2015). O que faz com que a sua manifestação adquira variações particulares em contextos culturais diferentes (BAEK; BULLOCK, 2014). Um complexo e dinâmico fenômeno psicossocial, cujo espaço de criação e desenvolvimento é a vida em sociedade, adicionado ao uso inadequado das tecnologias e que nos permite perceber que a tecnologia não é o ponto fulcral do problema. Ao contrário, a sociedade é que dá forma 
a tecnologia de acordo com as necessidades, valores e interesses das pessoas que utilizam as tecnologias (CASTELLS, 2006).

O cyberbullying pode ser visto como sintoma de uma violência social que apela ao nosso olhar e à nossa denúncia, para ser transformado em outra coisa. É preciso uma visão esclarecida, complexa, e, sobretudo, sistêmica (SOUZA et al., 2014a). Reforçamos a importância de políticas educativas no ensino superior que sejam promotoras da convivência social e ética, que sejam capazes de contribuir com ambientes acadêmicos de acolhida, valorização, apoio, reconhecimento das potencialidades e respeito à diversidade, facilitando a criação de climas de relações interpessoais positivas e promotoras da saúde e bem-estar psicossocial. Ações que fortaleçam as condições psicoemocionais e cognitivas dos estudantes, necessárias o bem-estar em contexto universitário (MASCARENHAS; MARTINEZ, 2012). Assim, esperamos ter contribuído para esse conhecimento partindo das experiências no terreno e ouvindo os estudantes que direta ou indiretamente estão envolvidos em situações de cyberbullying.

Agradecimento: Agradecemos a Fundação CAPES (Centro de Aperfeiçoamento de Pessoal de Nível Superior) pelo financiamento concedido através Programa de Doutorado Pleno no Exterior (BEX 1710/13-3).

\section{REFERÊNCIAS}

ABOUJAOUdE, E.; SAVAGE, M. W.; STARCEVIC, V.; SAlAME, W. O. Cyberbullying: review of an old problem gone viral. Journal of Adolescent Health, v.57, n.1, p.10-18, 2015.

AZEVEDO, P. Cyberbullying no ensino superior politécnico. (Dissertação de mestrado não publicada). Portalegre: Escola Superior de Educação do Instituto Politécnico de Portalegre, 2013.

BAEK, J.; BULLOCK, L. M. Cyberbullying: a cross-cultural perspective.Emotional and behavioural difficulties, v.19, n.2, p.226-238, 2014.

BAHIA, S.; FREIRE, I.; AMARAL, A.; ESTRELA, M. T. The emotional dimension of teaching in a group of Portuguese teachers. Teachers and Teaching, v.19, n.3, p.275$292,2013$.

BERKENBROCK-ROSITO, M. M.; JOSÉ, A. P. M. A estética do espaço escolar: narrativas discentes.Curitiba: CRV Editora, 2015.

CAETANO, A. P.; FREIRE, I.; VEIGA SIMÃO, A. M.; MARTINS, M. J. D.; 
PESSOA, M. T. Emoções no cyberbullying: um estudo com adolescentes portugueses. Revista Educação e Pesquisa, v.42, n.1, p.199-212, 2016.

CAPPADOCIA, M. C.; CRAIG, W. M.; PEPLER, D. Cyberbullying prevalence, stability, and risk factors during adolescence. Canadian Journal of School Psychology, v.28, n.2, p.171-192, 2013.

CASTAÑO, E.; BARCO, B. L. Estrategias de afrontamientodelestrés y estilos de conductainterpersonal. International Journal of psychology and psychological therapy, v. 10, n. 2, p.245-257, 2010.

CASTELlS, M. A Sociedade em Rede: do Conhecimento à Política. In Castells, M., \& Cardoso, G. (orgs.). A Sociedade em Rede: do Conhecimento à Acção Política Lisboa: Debates, Presidência da República, pp.17-30, 2006.

CASTILHO, A. E. Estudio descriptivo de las estratégias de afrontamientodelbullying, en professorado mexicano. Electronic Journal of Research in Educational Phychology, v.8, n.1, p.353-372, 2010.

CRYSTAL, D. S. Concepts of deviance in children and adolescents: The case of Japan. Deviant Behavior, v.15, n.3, p.241-266, 1994.

DILMAC, B. Psychological Needs as a Predictor of Cyber Bullying: A Preliminary Report on College Students. Educational Sciences: Theory and Practice, v.9, n.3, p.1307-1325, 2009.

DOANE, A. N.; PEARSON, M. R.; KELLEY, M. L. Predictors of cyberbullying perpetration among college students: An application of the Theory of Reasoned Action. Computers in Human Behavior, v.36, p. 154-162, 2014.

FAUCHER, C.; JACKSON, M.; CASSIDY, W. Cyberbullying among university students: Gendered experiences, impacts, and perspectives. Education Research International, v. 2014,p.1-10, 2014.

FRANCISCO, S. M. Cyberbullying: A faceta de um fenómeno em jovens universitários portugueses. (Dissertação de mestrado não publicada). Faculdade de Psicologia - Universidade de Lisboa, Lisboa, Portugal, 2012.

FRANCISCO, S. M.; VEIGA SIMÃO, A. M. V.; FERREIRA, P. C.; MARTINS, M. J. D. Cyberbullying: The hidden side of college students. Computers in Human Behavior, v.43, p.167-182, 2015.

FERREIRA, A. S.; VEIGA SIMÃO, A. M.; FERREIRA, A. I.; SOUZA, S. B.; FRANCISCO, S. M. Student bystander behavior and cultural issues in cyberbullying: When actions speak louder than words. Computers in Human Behavior, v.60,p.301$311,2016$.

FORBES, G.; ZHANG, X.; DOROSZEWICZ, K.; HAAS, K. Relationships between individualism-collectivism, gender, and direct or indirect aggression: A study in China, Poland, and the US. Aggressive Behavior, v. 35, n. 1, p.24-30, 2009.

GARAIGORDOBIL, M. Prevalencia y consecuencias del cyberbullying: 
unarevisión. International Journal of Psychology and Psychological Therapy, v. 11, n. 2, p.233 - 254, 2011.

GARAIGORDOBIL, M. Cyberbullying in adolescents and youth in the Basque Country: prevalence of cybervictims, cyberaggressors, and cyberobservers. Journal of Youth Studies, v. 18, n. 5, p.569-582, 2015.

GUALDO, A. M. G.; HUNTER, S. C.; DURKIN, K.; ARNAIZ, P.; MAQUILÓN, J. J. The emotional impact of cyberbullying: Differences in perceptions and experiences as a function of role. Computers \& Education, v. 82, p.228-235, 2015.

HINDUJA, S.; PATCHIN, J. Offline consequences of online victimization: School violence and delinquency. Journal of School Violence, v. 6, n. 3, p.89-112, 2007.

HOFSTEDE, G. Culture's consequences: Comparing values, behaviors, institutions, and organizations across nations ( $2^{\text {nd }}$ ed.). Thousand Oaks, CA: SAGE Publications, 2001.

LAZARUS, R. S. Cognition and motivation in emotion. American Psychologist, v. 46, n. 4, p.352 -367, 1991.

LI, Q. A cross-cultural comparison of adolescents' experience related to cyberbullying. Educational Research, v. 50, n. 3, p. 223-234, 2008.

LINDSAY, M., \& KRYSIK, J. Online harassment among college students. Information, Communication \&Society, v. 15, n. 5, p.703-719, 2012.

NA, H.; DANCY, B. L.; PARK, C. College student engaging in cyberbullying victimization: cognitive appraisals, coping strategies, and psychological adjustments. Archives of Psychiatric Nursing, v. 29, n. 3, p.155-161, 2015.

MARTINS, C. Manual de análise de dados quantitativos com recurso ao IBM SPSS: Saber decidir, fazer, interpretar e redigir. Braga: Psiquilíbrios Edições, 2011.

MARTINS, M. J. D. Maus tratos entre adolescentes na escola. Penafiel: Editora Novembro, 2009.

MASCARENHAS,S. A. N.; Martinez, J. M. A. Ocorrência do bullying/cyberbulling como desrespeito à diversidade e à cidadania no contexto universitário amazônico. Revista EDUCAmazônia, v. 8, n. 1, p.150-161, 2012.

OLWEUS, D. Cyberbullying: An overratedphenomenon? European Journal of Developmental Psychology, v. 9, n. 5, p.520-538, 2012.

ORTEGA, R.; ELIPE, P.; MORA-MERCHÁN, J. A.; GENTA, M. L.; BRIGHI, A.; GUARINI, A.; ...TIPPETT, N. The emotional impact of bullying and cyberbullying on victims: a European cross-national study. Aggressive Behavior, v. 38, n. 5, p.342-356, 2012.

PRICE, M.; DALGLEISH, J. Cyberbullying: Experiences, impact and coping strategies as described by Australian young people. Youth Studies Australia, v. 29, n. 2, p.51-59, 2010. 
SCHENK, A. M.; FREMOUW, W. J. Prevalence, psychological impact, and coping of cyberbully victims among college students. Journal of School Violence, v.11, n.1, p.21-37, 2012.

ŠEVČÍKOVÁ, A.; MACHÁČKOVÁ, H.; WRIGHT, M. F.; DĚDKOVÁ, L.; ČERNÁ, A. Social Support Seeking in Relation to Parental Attachment and Peer Relationships Among Victims of Cyberbullying. Journal of Psychologists and Counsellors in Schools, v. 25, n. 2, p.170-182, 2015.

SMITH, P. K.; MAHDAVI, J.; ARVALHO, M.; FISHER, S.; RUSSELL, S.; TIPPETT, N. Cyberbullying: Its nature and impact in secondary school pupils. Journal of Child Psychology and Psychiatry, v. 49, p.376-385, 2008.

SOUZA, S. B. Cyberbullying: a violência virtu@I conectada ao mundo real dos estudantes em contextos universitários do Brasil e Portugal. (Tese de doutoramento não publicada). Faculdade de Psicologia - Universidade de Lisboa, Lisboa, Portugal, 2016.

SOUZA, S. B. Cyberbullying: Estudo exploratório sobre as perspectivas acerca do fenómeno e das estratégias de enfrentamento com jovens universitários portugueses. (Dissertação de mestrado não publicada). Faculdade de Psicologia Universidade de Lisboa, Lisboa, Portugal, 2011.

SOUZA, S. B., VEIGA SIMÃO, A. M.; CAETANO, A. P. Cyberbullying: Percepções acerca do Fenômeno e das Estratégias de Enfrentamento. Psicologia: Reflexão e Crítica, v. 27, n. 3, p.582-590, 2014a.

SOUZA, S. B.; VEIGA SIMÃO, A. M.; FRANCISCO, S. M. Cyberbullying: incidência, consequências e contributos para o diagnóstico no ensino superior. Revista @ mbienteeducação, v. 7, n. 1, p.90-104, 2014b.

PESSOA, T.; MATOS, A.; AMADO, J.; JÄGER, T. Cyberbullying - do diagnóstico de necessidades à construção de um manual de formação. Revista Interuniversitaria de Pedagogía Social, v. 18, p.57-70, 2011.

WASHINGTON, E. T. An Overview of Cyberbullying in Higher Education. Adult Learning, v. 26, n. 1, p.21-27, 2015.

WILTON, C.; CAMPBELL, M. A. An exploration of the reasons why adolescents engage in traditional and cyberbullying. Journal of Educational Sciences \& Psychology, v. 1, n. 2, p.101 - 109, 2011.

YBARRA, M. L.; MITCHELL, K. J. Online aggressor/targets, aggressors, and targets: A comparison of associated youth characteristics. Journal of child Psychology and Psychiatry, v. 45, n. 7, p.1308-1316, 2004. 


\section{Como referenciar este artigo}

SOUZA, Sidclay Bezerra de, et al. O cyberbullying em contexto universitário do Brasil e Portugal: vitimização, emoções associadas e estratégias de enfrentamento. Revista Ibero-Americana de Estudos em Educação, Araraquara/SP, v. 11, n. esp. 3, p.16741691, 2016. Disponível em: 〈http://dx.doi.org/10.21723/riaee.v11.n.esp3.9067>. EISSN: 1982-5587.

Submetido em: agosto/2016

Aprovado em: novembro/2016 